\title{
Aplicación del estándar ISO/IEC 9126-3 en el modelo de datos conceptual entidad-relación
}

\author{
Standard ISO/IEC 9126-3 application in the entitu-relationship \\ conceptual data model
}

\section{Resumen}

Presenta una reflexión sobre las consideraciones y recomendaciones que se deben tener en cuenta para evaluar los criterios de calidad del Modelo Conceptual, dentro del ciclo de vida de las bases de datos, presentando una revisión de la temática, en la que se tienen en cuenta diversos factores dados por autores destacados en el área, y por estándares como la ISO/IEC 25012, la ISO/IEC 9126-3 y la ISO/IEC 25024.

Palabras clave: Modelo de Datos Conceptual, Modelo Entidad-Relación, ISO/IEC 9126-3, ISO/ IEC 25012, ISO/IEC 25024, Calidad en Bases de Datos.

\begin{abstract}
This paper presents a reflection about the considerations and recommendations to be taken into account, in order to evaluate the Conceptual Model's quality criteria, within the databases lifecycle; by introducing a thematic review, in which several factors are taken into account, like those given by the area renown authors and by the standards such as the ISO/IEC 25012, the ISO/ IEC 9126-3 and the ISO/IEC 25024.
\end{abstract}

Keywords: Conceptual Data Model, EntityRelationship Model, ISO/IEC 9126-3, ISO/IEC 25012, ISO/IEC 25024, Databases Quality.

\footnotetext{
* Heinsohn Business Technology (Colombia).

** Universidad Pedagógica y Tecnológica de Colombia (Colombia). juansebastian.gonzalez@uptc.edu.co
} 


\section{INTRODUCCIÓN}

Cuando se construye un software se suelen aplicar estándares de calidad para determinar el grado en que satisface las necesidades de los usuarios; sin embargo, en ocasiones se incurre en el error de considerar que si cumple con los factores dados por el estándar se asegura calidad, omitiendo la revisión de calidad en aspectos del desarrollo de software, como, por ejemplo, en el modelo conceptual de la base de datos; por tal razón, a lo largo de los años han surgido modelos y normas que permiten evaluar el grado en que estos modelos corresponden con los datos que desean almacenar las personas; no obstante, los estándares se encuentran aún en desarrollo, por lo que es necesario combinar normas enfocadas en calidad de datos y modelos de calidad de software. En este trabajo de investigación se plantea una guía que pueda ser aplicada para evaluar el grado de calidad del modelo conceptual durante el ciclo de vida de la base de datos.

El presente artículo describe inicialmente una serie de criterios que deben cumplir los modelos conceptuales, para contextualizar posteriormente sobre los estándares internacionales que han sido adoptados para la evaluación de calidad del modelo conceptual de datos, enfocándose principalmente en el estándar ISO/IEC 91263. A continuación de los fundamentos teóricos se presentan unos tópicos y recomendaciones, especificando los pasos que se deben seguir para realizar un correcto modelo conceptual. Finalmente, se presentan las conclusiones del proyecto de investigación y los trabajos que se derivan de esta investigación.

\section{Fundamentos teóricos}

Con el objetivo de generar mayor comprensión, es necesario tener presentes algunas definiciones, principalmente en cuanto a los criterios y estándares de calidad existentes para los modelos conceptuales.

\section{A. Criterios de calidad en modelos conceptuales}

Algunos autores han definido a lo largo de los años una serie de criterios de calidad que deben presentar los modelos conceptuales de bases de datos; el presente trabajo se limita a la notación Entidad-Relación (ER), debido a que es la de mayor uso a nivel mundial para el modelado de bases de datos relacionales [1-2]. En la Tabla 1 se describen los criterios que cabe resaltar.

\section{TABLA 1}

\section{CRiterios de CALIDAD EN MODELOS CONCEPTUALES}

\begin{tabular}{|l|l|}
\hline \multicolumn{1}{|c|}{ CRITERIO } & \multicolumn{1}{c|}{ DESCRIPCIÓN } \\
\hline LEGIBILIDAD & $\begin{array}{l}\text { Está enfocado a las consideraciones visuales para la lectura y presentación del } \\
\text { modelo conceptual (ausencia de cruces entre las relaciones, superposiciones, } \\
\text { tipografía clara, entre otros). }\end{array}$ \\
\hline COMPLETITUD & $\begin{array}{l}\text { El modelo debe incluir totalmente lo que se quiere diseñar, que es aquello que se } \\
\text { encuentra plasmado en los requerimientos del sistema por desarrollar. En términos } \\
\text { generales, cada requerimiento debe ser representado en el modelo. Y el modelo no } \\
\text { debe incluir requerimientos supuestos. }\end{array}$ \\
\hline CORRECCIÓN & $\begin{array}{l}\text { Se puede evaluar desde dos perspectivas: } \\
\text { La sintáctica, cuando las distintas partes de un modelo están construidas con } \\
\text { respecto al lenguaje utilizado, } \\
\text { y la semántica, cada elemento del problema se representa haciendo uso de las } \\
\text { estructuras adecuadas. }\end{array}$ \\
\hline
\end{tabular}




\begin{tabular}{|l|l|}
\hline \multicolumn{1}{|c|}{ CRITERIO } & \multicolumn{1}{c|}{ DESCRIPCIÓN } \\
\hline MINIMALIDAD & $\begin{array}{l}\text { Un modelo conceptual se considera mínimo si no tiene información redundante o } \\
\text { duplicada, y, por consiguiente, si se elimina un elemento del esquema se perderá } \\
\text { información. }\end{array}$ \\
\hline EXPRESIVIDAD & $\begin{array}{l}\text { El modelo representa la realidad, de manera que con sus elementos esta puede } \\
\text { ser comprendida fácilmente. La expresividad intenta medir la capacidad de } \\
\text { comunicación del modelo a nivel semántico. }\end{array}$ \\
\hline AUTOEXPLICACIÓN & $\begin{array}{l}\text { En el modelo pueden ser representados todos los requisitos, por consiguiente, la } \\
\text { lógica del negocio con respecto a los datos puede ser accedida y entendida por el } \\
\text { modelo conceptual. }\end{array}$ \\
\hline EXTENSIBILIDAD & $\begin{array}{l}\text { Se refiere a la capacidad de un esquema para poder tolerar cambios en los requisitos } \\
\text { y adaptarse a nuevas necesidades de los usuarios de la base de datos, es decir, el } \\
\text { esquema fácilmente se descompone en partes (módulos, vistas). }\end{array}$ \\
\hline
\end{tabular}

Basado en [3]

\section{B. Estándares de calidad en bases de datos}

1) ISO/IEC 25012: La división ISO/IEC 2501n, de la serie SQuaRe, presenta detalladamente los modelos de calidad para software y datos. En el estándar ISO/IEC 25012, que forma parte de esta división, se establecen las características externas de calidad, que a su vez se descomponen en subcaracterísticas deseables tanto en el software como en los datos, y se proporcionan consejos prácticos sobre el uso de modelos de calidad, formulando los lineamientos para la calidad de los datos almacenados en un sistema de información con un formato estructurado [4].

El estándar presenta quince características de calidad que pueden ser abordadas desde los puntos de vista dependiente e inherente del sistema:

- Dependiente del sistema: se refiere a cuando la calidad de los datos depende del dominio tecnológico en el que se hace uso de los datos [5]; en la Tabla 2 se presentan dichas características.

TABLA 2

\section{CARACTERÍSTICAS DEPENDIENTES SEGÚN ISO/IEC 25012}

\begin{tabular}{|l|l|}
\hline \multicolumn{1}{|c|}{ DIMENSIÓN } & \multicolumn{1}{c|}{ DESCRIPCIÓN } \\
\hline $\begin{array}{l}\text { Disponibilidad } \\
\text { (Availability) }\end{array}$ & $\begin{array}{l}\text { El grado en el cual el dato tiene atributos que le permiten ser recuperados por usuarios } \\
\text { autorizados o por aplicaciones en un contexto específico de uso. }\end{array}$ \\
\hline Portabilidad (Portability) & $\begin{array}{l}\text { El grado en el cual el dato tiene los atributos que le permiten ser instalado, substituido o } \\
\text { movido de un sistema a otro conservando la calidad existente en un contexto específico } \\
\text { de uso. }\end{array}$ \\
\hline $\begin{array}{l}\text { Recuperabilidad } \\
\text { (Recoverability) }\end{array}$ & $\begin{array}{l}\text { El grado en el cual el dato puede mantener y conservar un nivel especificado de operaciones } \\
\text { y calidad, aún en caso de falla. }\end{array}$ \\
\hline
\end{tabular}

Adaptado de [6]

- Inherente: "cuando la calidad de los datos se refiere a los datos en sí mismos; en general, este punto de vista está relacionado con los aspectos del dominio gestionados por los expertos" [5]; la descripción de estas características se presenta en la Tabla 3. 


\section{TABLA 3}

\section{CARACTERÍSTICAS INHERENTES SEGÚN ISO/IEC 25012}

\begin{tabular}{|l|l|}
\hline \multicolumn{1}{|c|}{ DIMENSIÓN } & \multicolumn{1}{c|}{ DESCRIPCIÓN } \\
\hline Exactitud (Accuracy) & $\begin{array}{l}\text { El grado en el cual el dato tiene atributos que representan correctamente el valor del } \\
\text { atributo intencionado de un concepto o evento en un contexto específico de empleo. }\end{array}$ \\
\hline $\begin{array}{l}\text { Completitud } \\
\text { (Completeness) }\end{array}$ & $\begin{array}{l}\text { El grado al cual el dato del sujeto asociado con una entidad tiene valores para todos los } \\
\text { atributos esperados e instancias de entidad relacionadas en un contexto específico de uso. }\end{array}$ \\
\hline $\begin{array}{l}\text { Consistencia } \\
\text { (Consistency) }\end{array}$ & $\begin{array}{l}\text { El grado en el cual el dato tiene los atributos que son libres de contradicción y son } \\
\text { coherentes con otros datos en un contexto específico de uso. }\end{array}$ \\
\hline Credibilidad (Credibility) & $\begin{array}{l}\text { El grado en el cual el dato tiene atributos que son considerados verdaderos y creíbles por } \\
\text { usuarios en un contexto específico de uso. }\end{array}$ \\
\hline Actualidad (Currentness) & $\begin{array}{l}\text { El grado en el cual el dato tiene los atributos que son del período correcto en un contexto } \\
\text { específico de uso. }\end{array}$ \\
\hline
\end{tabular}

Adaptado de [6]

En la Tabla 4 se presentan unas características comunes a ambas perspectivas. Además, el estándar también define un modelo de proceso [4] que puede ser aplicado para el uso del modelo de calidad de datos.

\section{TABLA 4}

\section{CARACTERÍSTICAS COMPARTIDAS SEGÚN ISO/IEC 25012}

\begin{tabular}{|l|l|}
\hline \multicolumn{1}{|c|}{ DIMENSIÓN } & \multicolumn{1}{c|}{ DESCRIPCIÓN } \\
\hline $\begin{array}{l}\text { Accesibilidad } \\
\text { (Accessibility) }\end{array}$ & $\begin{array}{l}\text { El grado en el cual el dato puede ser accesado en un contexto específico de uso, en particular } \\
\text { por la gente que necesita el soporte de tecnología o una configuración especial debido a } \\
\text { alguna inhabilidad (incapacidad). }\end{array}$ \\
\hline $\begin{array}{l}\text { Conformidad } \\
\text { (Compliance) }\end{array}$ & $\begin{array}{l}\text { El grado en el cual el dato tiene atributos que se adhieren a normas, convenciones o } \\
\text { regulaciones vigentes y reglas similares relacionadas con la calidad de datos en un contexto } \\
\text { específico de uso. }\end{array}$ \\
\hline $\begin{array}{l}\text { Confidencialidad } \\
\text { (Confidentiality) }\end{array}$ & $\begin{array}{l}\text { El grado en el cual el dato tiene los atributos que aseguran que solo es accesible e } \\
\text { interpretable por usuarios autorizados en un contexto específico de uso. }\end{array}$ \\
\hline Eficiencia (Efficiency) & $\begin{array}{l}\text { El grado en el cual el dato tiene los atributos que pueden ser procesados, y proporciona } \\
\text { los niveles esperados de funcionamiento (desempeño) usando las cantidades y los tipos de } \\
\text { recursos apropiados en un contexto específico de uso. }\end{array}$ \\
\hline Precisión (Precision) & $\begin{array}{l}\text { El grado en el cual el dato tiene atributos que son exactos o que proporcionan la } \\
\text { discriminación en un contexto específico de uso. }\end{array}$ \\
\hline $\begin{array}{l}\text { Trazabilidad } \\
\text { (Traceability) }\end{array}$ & $\begin{array}{l}\text { El grado en el cual el dato tiene atributos que proporcionan un rastro de auditoría de acceso } \\
\text { a los datos y de cualquier cambio hecho a los datos en un contexto específico de uso. }\end{array}$ \\
\hline $\begin{array}{l}\text { Entendibilidad } \\
\text { (Understandability) }\end{array}$ & $\begin{array}{l}\text { El grado en el cual el dato tiene atributos que le permiten ser leído e interpretado por } \\
\text { usuarios, y es expresado en lenguajes apropiados, símbolos y unidades en un contexto } \\
\text { específico de uso. }\end{array}$ \\
\hline
\end{tabular}

Adaptado de [6]

Algunos investigadores han aplicado el ISO/IEC 25012 para determinar la calidad de información y datos referentes a las aplicaciones desarrolladas; además de las anteriores características se han agregado dos nuevas que, aunque no forman parte del estándar, pueden ser tenidas en cuenta para la 
elaboración de la guía. La primera de ellas es la Adecuación Representacional (Representational adequacy), definida como la medida en que los datos se representan en forma concisa, flexible y organizada de acuerdo con su nivel de relevancia; la segunda es el valor agregado (value added), que consiste en la medida en que la utilización de los datos proporcionan ventajas; la razón de la adición de esta característica es que los datos pueden ser completos, fiables y actualizados, pero, sin embargo, no ofrecer beneficio alguno a los usuarios [7].
2) ISO/IEC 9126 -3: Esta parte del estándar ISO/IEC 9126 es un reporte técnico que incluye las métricas internas que se pueden aplicar a un producto de software; cabe destacar que al ser métricas internas se aplican a productos de software no ejecutables; además, presenta una serie de ejemplos sobre métricas que pueden ser aplicadas y un marco de trabajo (framework) para realizar mediciones a un producto de software particular [8]. En la Tabla 5 se ilustra la equivalencia entre las características del ISO/IEC 9126 y el ISO/IEC 25012, que actualmente se usa como base para adaptarlo al modelo de calidad de datos del ISO 25012.

TABLA 5

CORRESPONDENCIA ENTRE LAS CARACTERÍSTICAS DEL ISO 9126 E ISO/IEC 25012

\begin{tabular}{|c|c|c|}
\hline \multicolumn{2}{|r|}{ ISO 9126} & \multirow{2}{*}{$\begin{array}{c}\text { ISO/IEC 25012 } \\
\text { CARACTERÍSTICA }\end{array}$} \\
\hline CARACTERÍSTICA & SUBCARACTERÍSTICA & \\
\hline \multirow{4}{*}{ Funcionalidad } & Idoneidad & $\begin{array}{l}\text { Consistencia } \\
\text { Actualidad } \\
\text { Completitud } \\
\text { Precisión }\end{array}$ \\
\hline & Exactitud & Exactitud \\
\hline & Interoperatibilidad & \\
\hline & Seguridad & Seguridad \\
\hline \multirow{4}{*}{ Fiabilidad } & & Disponibilidad \\
\hline & Madurez & \\
\hline & Tolerancia a fallos & \multirow{2}{*}{ Recuperabilidad } \\
\hline & Facilidad de recuperación & \\
\hline \multirow[b]{3}{*}{ Usabilidad } & Facilidad de comprensión & Entendibilidad \\
\hline & Facilidad de aprendizaje & \\
\hline & Operatividad & $\begin{array}{l}\text { Accesibilidad } \\
\text { Manejabilidad }\end{array}$ \\
\hline Eficiencia & $\begin{array}{l}\text { Tiempo de uso } \\
\text { Recursos utilizados }\end{array}$ & Eficiencia \\
\hline \multirow{3}{*}{ Mantenibilidad } & Facilidad de análisis & \\
\hline & Facilidad de cambio & Facilidad de cambio \\
\hline & $\begin{array}{l}\text { Estabilidad } \\
\text { Facilidad de prueba }\end{array}$ & \\
\hline Portabilidad & $\begin{array}{l}\text { Facilidad de instalación } \\
\text { Facilidad de ajuste } \\
\text { Facilidad de adaptación al cambio }\end{array}$ & Portabilidad \\
\hline
\end{tabular}


3) ISO/IEC 25024: Está aún en desarrollo, y se espera que trabaje en conjunto con el ISO/IEC 25012, debido a que fue diseñado con el fin de proporcionar medidas orientadas netamente a la calidad de datos [9].

\section{Aplicación de MÉTRICAS DE CALIDAD A MODELOS CONCEPTUALES DE DATOS}

El modelo conceptual es el enlace entre los requisitos funcionales de un sistema de información y el diseño de la base de datos, lo que implica una serie de actividades que permitan definir qué se va a representar y cómo se va a representar, independientemente del gestor de bases de datos que se vaya a utilizar; hay que tener en cuenta que existen restricciones del mundo real que no pueden ser representadas en un modelo conceptual, por lo que es necesario adicionar representaciones textuales al diagrama [10].

La primera tarea es especificar los requisitos de la aplicación en un lenguaje natural; para esto es necesario realizar encuestas y entrevistas a las personas que están involucradas en la organización o empresa, revisar la documentación usada $\mathrm{y}$, si existe algún software, detallar las interfaces y formularios que son manejados por él; esto puede consignarse en un documento de especificación de requisitos, que representa un esquema percibido de lo que se va a representar.

La segunda tarea consiste en obtener el diagrama conceptual a partir de los requisitos; para esto, algunos autores nos presentan unas claves para identificar entidades, atributos y relaciones:

- Entidades: serán identificadas de los requisitos como objetos reales o abstractos de los cuales se deseaalmacenar información [11]; suelen estar en forma de sustantivos. Las entidades se caracterizan por poder ser descritas o descompuestas en elementos más pequeños; además, existen dos tipos de entidades: las fuertes, que pueden existir por sí mismas, es decir, sin dependencia de otra entidad, y las débiles, cuya existencia depende de la ocurrencia de otra entidad y, por consiguiente, si se elimina la ocurrencia de la entidad fuerte se elimina su existencia.

- Atributos: pueden ser obtenidos de los requisitos como propiedades o características que tiene una entidad [11]. Los atributos suelen estar en singular y son indivisibles, si un atributo puede ser descompuesto es indicio de que es una entidad o puede acabar como una relación, porque se haya determinado que el atributo es una referencia a otro tipo de entidad [12].

- Relaciones: pueden obtenerse a partir de los verbos que interactúan con dos o más sustantivos (libro alquilado por un estudiante), las relaciones son asociaciones o correspondencias entre entidades, además, las relaciones presentan cierto grado de cardinalidad con las que cada tipo de entidad interviene en el tipo de interrelación [11].

Una vez que se han identificado los anteriores elementos y se diagraman acorde con una notación particular, se debe buscar que el modelo conceptual [13] se caracterice por su:

- Claridad, esto es, que la significación no sea ambigua

- Coherencia, es decir, no existan contradicciones o confusiones

- Plenitud, en cuanto a que el esquema representa lo esencial del fenómeno

- Fidelidad, en el sentido de que la representación del universo del discurso ha de hacerse $\sin$ desviaciones $\mathrm{ni}$ deformaciones

- Simplicidad, pues se ha de buscar la máxima sencillez 
También puede aplicar una serie de Reglas de consistencia e integridad [14], como se muestran en la Tabla 6, que le permiten verificar si el modelo conceptual fue correctamente elaborado, y si no lo fue, proceder a hacer las respetivas correcciones.

\section{TABLA 6}

\section{REGLAS DE CONSISTENCIA E INTEGRIDAD}

\begin{tabular}{|c|c|}
\hline TIPO & DESCRIPCIÓN \\
\hline \multirow[t]{5}{*}{ Integridad } & $\begin{array}{l}\text { 1. Regla de la llave primaria: todos los tipos de entidades tiene una llave primaria (propia, } \\
\text { compuesta o heredada) }\end{array}$ \\
\hline & 2. Regla de la denominación: todos los tipos de entidad, relaciones y atributos tienen nombre. \\
\hline & 3. Regla de la cardinalidad: está dada para los dos grados que pueden existir en una relación. \\
\hline & $\begin{array}{l}\text { 4. Regla de la participación de la entidad: todos los tipos de entidad participan en al menos } \\
\text { una relación, excepto los de una jerarquía de generalización. }\end{array}$ \\
\hline & $\begin{array}{l}\text { 5. Regla de participación en una jerarquía de generalización: cada jerarquía de generalización } \\
\text { participa en al menos una relación con un tipo de entidad que no está en la jerarquía de } \\
\text { generalización. }\end{array}$ \\
\hline \multirow[t]{9}{*}{ Consistencia } & 1. Regla de nombres de entidad: los nombres de una entidad son únicos. \\
\hline & 2. Regla de nombre de atributo: los nombres de atributos son únicos dentro de las de entidades. \\
\hline & $\begin{array}{l}\text { 3. Regla de nombre de atributos heredados: los nombres de los atributos de un subtipo no } \\
\text { coinciden con los nombres de los atributos heredados (directos o indirectos) }\end{array}$ \\
\hline & $\begin{array}{l}\text { 4. Regla de tipo de conexión relación/entidad: todas las relaciones conectan dos tipos de } \\
\text { entidad (no necesariamente distintos). }\end{array}$ \\
\hline & 5. Regla de conexión relación/relación: las relaciones no se conectan con otras relaciones. \\
\hline & 6. Regla de entidad débil: las entidades débiles tienen al menos una relación identificable. \\
\hline & $\begin{array}{l}\text { 7. Regla de la relación identificable: para cada relación identificable al menos uno de los tipos } \\
\text { de entidad participante debe ser débil. }\end{array}$ \\
\hline & $\begin{array}{l}\text { 8. Regla de la Cardinalidad de identificación de dependencia: para cada relación identificable, } \\
\text { la cardinalidad mínima y máxima debe ser } 1 \text { en el sentido del tipo de entidad hijo (entidad } \\
\text { débil) al tipo de entidad padre. }\end{array}$ \\
\hline & 9. Regla de la llave foránea redundante: las llaves foráneas redundantes no se usan. \\
\hline
\end{tabular}

\section{A. Mediciones al Diagrama Entidad-Relación}

Para ejemplificar la medición al modelo, se usará el modelo conceptual presentado en la Fig. 1, basado en la lógica de negocio de una compraventa de vehículos. Una vez que se revisa el cumplimiento de las reglas de consistencia e integridad de la Tabla 7, se procede a seguir el marco de trabajo para la elaboración de las métricas de calidad basado en el estándar ISO/ IEC 9126-3. 


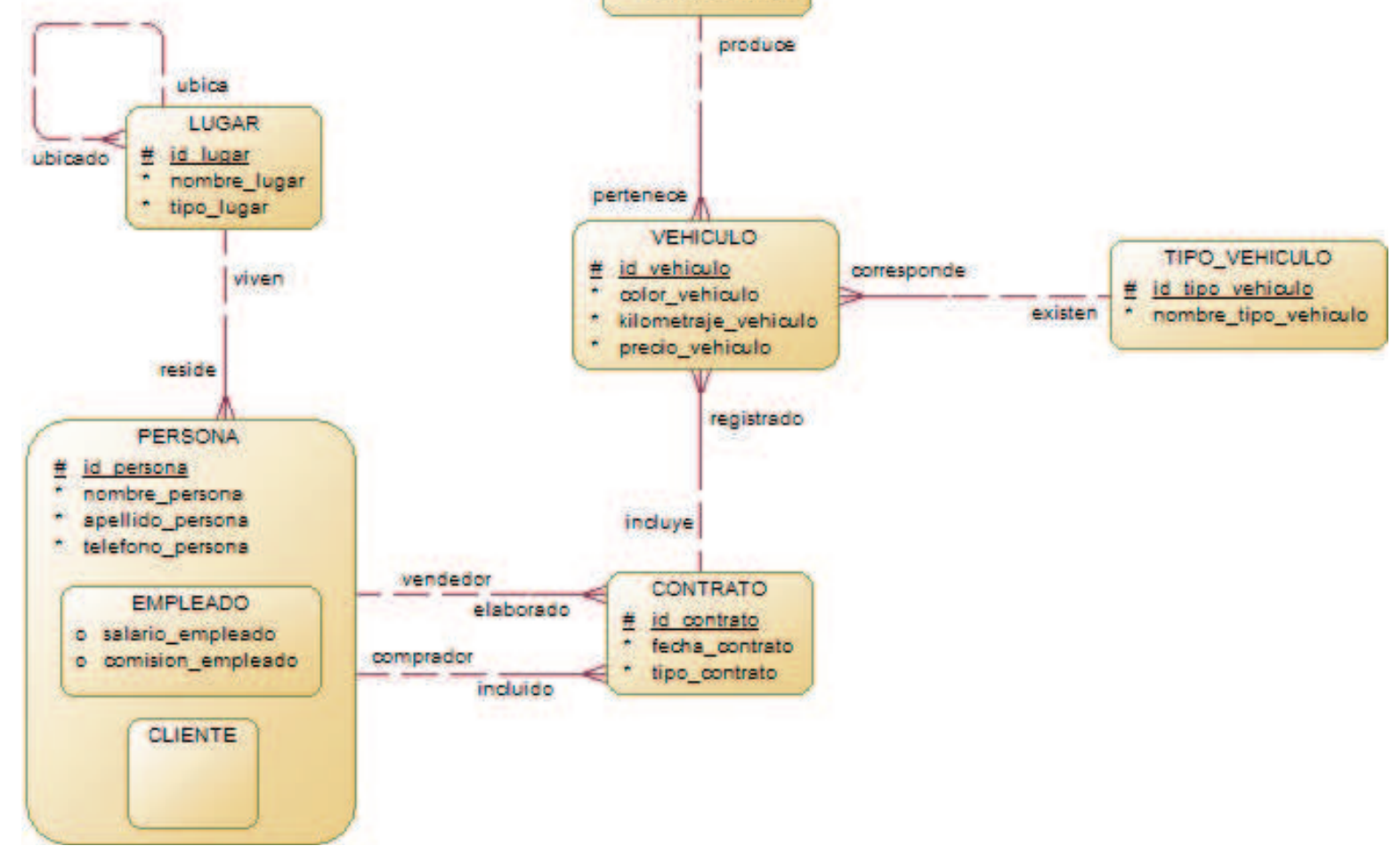

Fig. 1. Modelo Conceptual de compra-venta de vehículos

1) Identificación de requisitos de calidad: Los requisitos de calidad corresponden a las subcaracterísticas que se van a evaluar; cabe destacar que el modelo conceptual es un producto de software no ejecutable, por lo que se aplicarán métricas internas según ISO 9126. Además de lo anterior, hay una serie de criterios que no serán evaluados, como, por ejemplo, la portabilidad, debido a que es un criterio que requiere de una implementación física del modelo de datos; por lo que solo se evaluarán características que son susceptibles de ser aplicadas al modelo conceptual. En la Tabla 7 se resumen las características por evaluar y el peso que tendrá cada una; cabe aclarar que el peso está especificado de manera cualitativa, sin embargo, para efectos prácticos se utilizará una escala de $0 \mathrm{a} 1$, siendo repartidos los pesos de la siguiente manera:

Bajo (B): 0,00-0,33

Medio (M): 0,34-0,75

Alto (A): 0,76-1,00 
TABLA 7

IDENTIFICACIÓN DE LOS REQUISITOS DE CALIDAD DEL MODELO CONCEPTUAL

\begin{tabular}{|l|l|l|l|}
\hline CARACTERÍSTICA ISO 9126 & CARACTERÍSTICA ISO 25012 & DIMENSIÓN & PESO \\
\hline \multirow{4}{*}{ Funcionalidad } & Consistencia & I & A \\
& Completitud & I & A \\
& Precisión & I/D & A \\
& Exactitud & I & I \\
& Actualidad & I/D & B \\
\hline \multirow{2}{*}{ Usabilidad } & Entendibilidad & I/D & M \\
\hline Mantenibilidad & Manejabilidad & I & M \\
\hline
\end{tabular}

2) Diseño de la evaluación: En esta etapa se asocian a cada subcaracterística los objetos por evaluar; para el caso particular será exclusivamente el modelo conceptual, sin embargo, las fuentes de medición de algunas métricas incluyen el documento de especificación de requisitos. Adicionalmente, se codificó cada métrica interna con un código único para elaborar la Tabla 8 , donde fue necesario especificar las métricas por evaluar; en este caso se aplican exclusivamente métricas internas cuyos propósitos y métodos de aplicación fueron descritos anteriormente.

Ciertas características del ISO 25012 se evalúan con varias métricas diseñadas con base en el marco de trabajo de ISO 9126-3, pero modificadas a los criterios de calidad del modelo de calidad de datos; por otro lado, se tienen otras características, como la exactitud, que serán evaluadas por una sola métrica, debido a que para el caso particular se mide exclusivamente la exactitud de los atributos de dominio.

TABLA 8

PLAN DE MEDICIÓN

\begin{tabular}{|l|l|l|}
\hline \multicolumn{1}{|c|}{ CARACTERÍSTICA } & \multicolumn{1}{|c|}{ SUBCARACTERÍSTICA } & \multicolumn{1}{|c|}{ MÉTRICAS INTERNAS POR APLICAR } \\
\hline Funcionalidad & Consistencia & C1: Nombres de entidad \\
& & C2: Nombres de atributos heredados \\
& & C3: Conexión entidad/relación \\
& & C4: Conexión de entidades débiles \\
& C5: Adecuación funcional \\
& C6: Requisitos inexistentes \\
& Completitud & C7: Tipos de datos \\
& Precisión & P1: Atributos numéricos \\
& P2: Cadenas de caracteres \\
& Exactitud & Precisión de dominios \\
& E1: Exactitud de dominios \\
\hline Usabilidad & Entendidadilidad & U1: Cruce de relaciones \\
& & U2: Superposición de entidades \\
& Manejabilidad & U3: Tipografía clara \\
& M1: Correspondencia datos y atributos \\
\hline Mantenibilidad & Facilidad de cambio & R1: Registro de cambios \\
\hline
\end{tabular}


3) Especificación de la evaluación: Para cada característica de calidad definida en la Tabla 8 es necesario definir las métricas que van a ser aplicadas, así como los niveles de satisfacción que se consideran deben cumplir las ponderaciones para ser consideradas de calidad. En la Tabla 9 se presentan las fórmulas dadas para cada métrica a criterio del autor, con base en una serie de procedimientos diseñados acorde al marco de trabajo del ISO 9126-3.

TABLA 9

IDENTIFICACIÓN DE LOS REQUISITOS DE CALIDAD DEL MODELO CONCEPTUAL

\begin{tabular}{|c|c|c|c|}
\hline CARACTERÍSTICA & SUBCARACTERÍSTICA & MÉTRICA & $\begin{array}{c}\text { NIVEL } \\
\text { MÍNIMO } \\
\text { REQUERIDO }\end{array}$ \\
\hline Funcionalidad & $\begin{array}{l}\text { Consistencia } \\
\text { Completitud } \\
\text { Precisión } \\
\text { Exactitud } \\
\text { Actualidad }\end{array}$ & \begin{tabular}{|l|}
$(\mathrm{C} 1+\mathrm{C} 2+\mathrm{C} 3+\mathrm{C} 4) / 4$ \\
$(\mathrm{C} 5+\mathrm{C} 6+\mathrm{C} 7) / 3$ \\
$(\mathrm{P} 1+\mathrm{P} 2+\mathrm{P} 3) / 3$ \\
E1 \\
No aplica
\end{tabular} & $\begin{array}{l}0,95 \\
0,95 \\
0,90 \\
0,55 \\
---\end{array}$ \\
\hline Usabilidad & $\begin{array}{l}\text { Entendibilidad } \\
\text { Manejabilidad }\end{array}$ & $\begin{array}{l}(\mathrm{U} 1+\mathrm{U} 2+\mathrm{U} 3) / 3 \\
\mathrm{M} 1\end{array}$ & $\begin{array}{l}0,95 \\
0,55\end{array}$ \\
\hline Mantenibilidad & Facilidad de cambio & R1 & 0,70 \\
\hline
\end{tabular}

4) Evaluación y verificación de los criterios de calidad: En la Tabla 10 se detalla la especificación de la evaluación obtenida; cabe aclarar que la evaluación se efectuó con base en los requerimientos bajo los cuales se diseñó el modelo, los cuales no se presentan por la extensión del documento.

\section{Tabla 10}

RESULTADOS DE EVALUACIÓN DE MÉTRICAS

\begin{tabular}{|l|c|c|}
\hline \multicolumn{1}{|c|}{ MÉTRICA } & PUNTAJE & TOTAL CARACTERÍSTICA \\
\hline C1: Nombres de entidad & 0,92 & 0,98 \\
C2: Nombres de atributos heredados & 1 & \\
C3: Conexión entidad/relación & 1 & \\
C4: Conexión de entidades débiles & 1 & 0,93 \\
\hline C5: Adecuación funcional & 0,89 & \\
C6: Requisitos inexistentes & 0,91 & 0,95 \\
C7: Tipos de datos & 1 & \\
\hline P1: Atributos numéricos & 1 & 0,95 \\
P2: Cadenas de caracteres & 1 & 1 \\
P3: Precisión de dominios & 0,85 & \\
\hline E1: Exactitud de dominios & 0,95 & \\
\hline U1: Cruce de relaciones & 1 & 0,96 \\
U2: Superposición de entidades & 1 & 0 \\
U3: Tipografía clara & 1 & \\
\hline M1: Correspondencia datos y atributos & 0,96 & \\
\hline R1: Registro de cambios & 0 & \\
\hline
\end{tabular}

Una vez aplicada la evaluación de las métricas se procede a contrastar las columnas "Nivel Mínimo
Requerido" de la Tabla 9 y "Puntaje" de la Tabla 10; las métricas cuyos valores estén por debajo 
del nivel requerido corresponden a los elementos del modelo que necesitan ser modificados para posteriormente volver a realizar un análisis sobre ellos. En el caso de la característica de Mantenibilidad se asignó cero (0) al puntaje, debido a que el modelo no sufrió modificaciones en los requisitos, por consiguiente, no se puede realizar la proyección de facilidad de cambio del modelo; sin embargo, esto no quiere decir que el modelo no tenga calidad, sino que simplemente esta característica queda pendiente para una evaluación futura.

5) Transformaciones del modelo conceptual: Las transformaciones por realizar deben hacerse con base en los aspectos dados por las métricas cuyo puntaje no satisface los niveles requeridos; el caso de estudio se caracteriza por no estar aún completo, lo que ocasiona que no se tenga una adecuación funcional completa; esto indica que es necesario verificar los requisitos funcionales que aún no han sido modelados, y plasmarlos en el modelo. La existencia de requisitos inexistentes puede deberse al uso de atributos y entidades que no están estipuladas en los requisitos funcionales; así, pues, las transformaciones se pueden realizar con base en las siguientes actividades:

- Consistencia: reglas para nombrar atributos, entidades y relaciones, además de verificar el correcto uso de entidades débiles.

- Completitud: verificación de requisitos funcionales y de los objetos que los satisfacen en el modelo conceptual.

- Precisión: especificación de tipos de datos en los atributos y creación de dominios basados en la lógica del negocio.

- Exactitud: verificar que los dominios que fueron creados correspondan con los atributos del modelo conceptual consignados para tal fin.
- Entendibilidad: tener en cuenta las reglas de notación, ortografía y la disposición de los elementos del modelo conceptual.

- Manejabilidad: verificar la correspondencia de los atributos con los tipos de datos que les son asignados (no incluye atributos de dominio).

- Facilidad de cambio: si los requisitos funcionales han cambiado durante la elaboración del modelo es posible realizar una proyección para determinar si el diagrama conceptual se adaptará; sin embargo, esta característica es la más difícil de medir, debido a que se refiere a necesidades futuras cuyo impacto en el modelo conceptual es incierto.

\section{Conclusiones}

Las consideraciones y recomendaciones presentadas permiten evaluar la calidad de un modelo conceptual de la base de datos; aunque la manera de realizar esta tarea es subjetiva, se decidió trabajar con el marco de trabajo estipulado por el reporte técnico ISO 9126-3, de manera que se puedan validar las métricas creadas. Las mediciones absolutas que se utilizaron permiten realizar conteos precisos de los elementos del diagrama; con esto se tiene una alternativa al uso de ecuaciones y tratamientos matemáticos complejos.

Es fundamental trabajar de la mano con el documento de especificación de requisitos, debido a que ciertas métricas lo necesitan como fuente de medición; también es necesario haber terminado el modelo conceptual en su totalidad para evaluar la facilidad de cambio, pues esta solo permite establecer una proyección para determinar la adaptabilidad del modelo conceptual.

El uso de métricas en etapas tempranas, como la especificación y el diseño de la base de datos, aumenta la calidad no solo al modelo conceptual, 
sino también al software. Es indispensable trabajar con estándares de calidad enfocados en el modelo de datos (ISO/IEC 25012), de manera que las características por evaluar sean las correctas.

\section{RECOMENDACIONES Y TRABAJOS FUTUROS}

El presente trabajo fue diseñado para ser aplicado exclusivamente al modelo conceptual de datos. Independientemente de la notación usada, algunas métricas estipulan que se debe evaluar la calidad en cuanto a las normas de diagramación de dicha notación. Las métricas fueron diseñadas con base en los aspectos relacionados en el aparte de los fundamentos teóricos, y posteriormente fueron adaptadas a la tabla del estándar ISO/IEC 91263 ; esto con el fin de adecuar métricas de software al modelo conceptual de datos.

Debido a que el estándar ISO/IEC 25024 aún se encuentra en desarrollo, es necesario que se plantee continuar este trabajo para utilizar las métricas que sean estipuladas por la ISO, esto debido a que este estándar está siendo diseñado con el fin de aplicar netamente métricas orientadas al modelo de calidad de datos.

También se hace necesario ampliar el trabajo para evaluar la calidad de los modelos lógico y físico de la base de datos, con el fin de crear una guía que pueda ser aplicada a todo el ciclo de vida de las bases de datos y así fomentar su calidad.

\section{REFERENCIAS}

[1] V. Fernández. Desarrollo de sistemas de información: Una metodología basada en el modelado. España: Universidad Politécnica de Cataluña, 2010.

[2] O. D. Chávez. Modelo Entidad-Relación (Material de clase). Perú: Universidad Privada San Pedro, 2007.
[3] M. Varas y J. Pradenas. Hacia la definición de Métricas de Calidad para Esquemas Conceptuales de Bases de Datos [en línea]. Chile: Universidad de Concepción. Disponible en: http://www.inf.udec. $\mathrm{cl} / \sim$ mvaras/papers/2000/varas-pradenas. pdf

[4] Software engineering -Software product Quality Requirements and Evaluation (SQuaRE) - Data Quality Model, ISO/IEC 25012, 2008.

[5] C. Calero et al. Calidad del producto y proceso software. Ed. Ra-Ma, 2010.

[6] C. A. García. Calidad y Medición de Sistemas de Información, 2009.

[7] I. Rafique, P. Lew, M., Qanber Abbasi, and Z. Li. "Information Quality Evaluation Framework: Extending ISO 25012 Data Quality Model", World Academy of Science, Engineering and Technology, 65: 523-528, 2012.

[8] Software Engineering - Product quality Part 3: Internal metrics, ISO/IEC TR 91263, 2003.

[9] Systems and Software Quality Requirements and Evaluation (SQuaRE) - Measurement of data quality, ISO/IEC 25024 CD1, 2013.

[10] I. Luque, M. A. Gómez-Nieto, E. López, and G. Cerruela, Bases de Datos Desde Chen hasta Codd con ORACLE, Ed. RaMa., 2002, pp. 56-58.

[11] A.de M. Castañoy M. Piattini.Fundamentos $y$ modelos de Bases de Datos, Ra-Ma., 2001.

[12] R. Elmasri y S. B. Navathe. Fundamentos de Sistemas de Bases de Datos, Ed. Pearson Educación, 2007. 
[13] A. de M. Castaño y M. Piattini. Concepción y Diseño de Bases de Datos del Modelo E/R al Modelo Relacional, Ed. Ra-Ma., 1993.
[14] M. V. Mannino. Administración de bases de datos. Diseño y desarrollo de aplicaciones, McGraw-Hill Interamericana, 2007. 